\title{
PROPUESTA PARA EL CÁlCULO DEL ALFA ORDINAL Y THETA DE ARMOR
}

\author{
PROPOSAL FOR THE CALCULATION OF THE ORDINAL ALPHA AND ARMOR \\ THETA
}

Sergio Dominguez L. ${ }^{1}$

Universidad Nacional Mayor de San Marcos, Lima, Perù

(RECiBIDo 03/4/2012; AcEptAdo 15/6/2012)

\begin{abstract}
RESUMEN
Este artículo presenta un programa en formato MS Excel para el cálculo del coeficiente Alfa Ordinal y Theta de Armor, usado para cuantificar la confiabilidad en ítems de respuesta categórica ordenada. La construcción de los comandos fue hecha en base a la matriz de correlaciones policóricas resultantes del programa FACTOR (Lorenzo \& Ferrando, 2007). El uso adecuado de estos métodos y su falta de implementación en los paquetes estadísticos tradicionales justifica su construcción como una herramienta para el investigador en psicología, disciplina en la cual es común el uso de matrices de correlaciones de Pearson con las limitaciones que esta acarrea (asume que la variable estudiada es continua). Se discute el uso y las limitaciones de la herramienta.
\end{abstract}

Palabras clave: confiabilidad, alfa ordinal, theta de armor, matriz de correlaciones policóricas

\begin{abstract}
The present article presents a program in MS Excel format for the calculation of the coefficient Ordinal Alpha and Armor Theta, used to quantify the reliability in ordinal response items. The construction of the commandos was made based on the polychoric correlation matrix of the program FACTOR (Lorenzo \& Ferrando, 2007). The suitable use of these methods and their lack of implementation in traditional statistical packages justifies its construction like a tool for the investigator in psychology, discipline in which is common the use of Pearson correlation matrix with the limitations that this carries (it assumes that the studied variable is continuous). It is discussed the use and the limitations of the tool.
\end{abstract}

Keywords: reliability, ordinal alpha, armor theta, polychoric correlation matrix

1 Docente investigador de la Facultad de Psicología y Trabajo Social de la Universidad Inca Garcilaso de la Vega. Estudiante de Maestría en Psicología Clínica y de la Salud de la Facultad de Psicología de la Universidad Nacional Mayor de San Marcos. E-mail: sdominguezmpcs@gmail.com 


\section{CONFIABILIDAD: ASPECTOS CONCEPTUALES BÁSICOS}

Diversos autores han brindado su punto de vista acerca del concepto de confiabilidad. Algunos señalan que la confiabilidad es la proporción de la varianza total atribuida a la varianza verdadera, y en consecuencia, entre mayor sea la proporción de la varianza total atribuida a la varianza verdadera, la prueba será más confiable (Cohen \& Swerdlick, 2001; Alarcón, 1998). Por otro lado, otros autores, como Hogan (2004), mencionan que la confiabilidad se relaciona con la consistencia de las puntuaciones en la medición al margen de lo que mida el instrumento. Muñiz (1994) por su parte refiere que la confiabilidad se refiere a la estabilidad de las mediciones cuando no existan razones teóricas o empíricas para suponer que la variable a medir haya sido modificada diferencialmente para los sujetos. Otra definición la dan Prieto \& Delgado (2010), para quienes la fiabilidad se concibe como la consistencia o estabilidad de las medidas cuando el proceso de medición se repite. Se puede concluir parcialmente que la confiabilidad suele estar definida tanto en términos de constancia temporal como de su homogeneidad, es decir, si los reactivos que conforman la prueba son consistentes entre sí.

\section{EL ALFA DE CRONBACH}

Cuando se desea estimar la confiabilidad por medio de la consistencia temporal de las puntuaciones, se establece una correlación de las dos aplicaciones sobre las puntuaciones totales del test, sin importar cual es el formato de ítems que contiene la prueba, sea dicotómico o politómico, y si fuera de este último modo, con cuántas opciones de respuesta cuenta. En cambio, con la estimación de la confiabilidad por consistencia interna, el tipo de ítem cobra importancia, porque dependiendo el formato del mismo existen diversos estimadores, dependiendo básicamente de cuantas opciones de respuesta tenga.

Se tiene entonces que con ítems dicotómicos, es más aceptado el uso del estimador KR-20. Con ítems politómicos sobrevienen las dificultades. Es conocido que el uso del coeficiente Alfa de Cronbach es propuesto muchas veces sin considerar las restricciones que tiene su uso (Campo-Arias \& Oviedo, 2008; Oviedo \& Campo-Arias, 2005), y los problemas que podría acarrear en el caso que su uso sea inadecuado, como por ejemplo, en escalas de cuatro o menos opciones de respuesta (Lozano, García-Cueto \& Muñiz, 2008; Weng, 2004), debido a que es preferible porque solo se requiere una aplicación del instrumento para lograr su estimación y está disponible como la única opción para estimar la estabilidad por consistencia interna en diversos paquetes estadísticos comerciales. Sumado a ello, la limitación más seria que presenta el coeficiente Alfa de Cronbach, es que supone el carácter continuo de las variables, algo que no se cumple en ciencias sociales, lo que lleva a una infraestimación de la confiabilidad (Elosua \& Zumbo, 2008).

Elosua \& Zumbo (2008) realizaron una revisión sobre la pertinencia del uso de otros estimadores de la confiabilidad por consistencia interna para el caso de ítems que tengan menos de cinco opciones de respuesta, como es el caso del Alfa ordinal 
desde el modelo del factor común y el coeficiente Theta de Armor, desde el modelo de los componentes principales. Dichos autores plantean expresiones matemáticas para su cálculo, apoyados con los output de correlaciones policóricas del software libre FACTOR (Lorenzo \& Ferrando, 2007), mas no una herramienta que permita el cálculo directo utilizando los datos provenientes de dicho programa, sin recurrir a procedimientos manuales para hallar dichos coeficientes.

\section{PROPUESTA DE LA HERRAMIENTA Y SU DISPONIBILIDAD}

La propuesta del presente trabajo es una hoja de cálculo en formato de Microsoft Excel, compatible con todas las versiones de Windows. Está disponible al usuario sin costo escribiendo al autor del trabajo.

La herramienta está basada en el trabajo de Elosua \& Zumbo (2008), la cual permite el cálculo rápido tanto del Alfa ordinal como del coeficiente Theta de Armor, mediante la colocación de los datos necesarios para cada uno. Es decir, en el caso del Alfa ordinal, los pesos factoriales (correlación ítem-factor) derivados de la matriz policórica y el número de ítems que componen el factor (Tabla 1) para así obtener las comunalidades y unicidades de modo automático (Tabla 2) y así llegar a la estimación del alfa ordinal (Figura 1).

\section{CALCULO DE ALPHA ORDINAL}

COLOCA AQUí LOS PESOS FACTORALES DE CADA ITEM

(Usar datos basados en la matriz de correlaciones policóricas-FACTOR)

\begin{tabular}{|c|c|}
\hline ITEM & PESO FACTORIAL \\
\hline 1 & 0.859 \\
\hline 2 & 0.281 \\
\hline 3 & 0.625 \\
\hline 4 & 0.855 \\
\hline 5 & 0.481 \\
\hline 6 & 0.486 \\
\hline
\end{tabular}

Tabla 1. Presentación de módulo de cálculo del Alfa Ordinal

\begin{tabular}{|c|c|c|c|}
\hline ITEM & PESO FACTORIAL & COMUNALIDAD & UNIDAD \\
\hline 1 & 0.859 & 0.737881 & 0.262119 \\
\hline 2 & 0.281 & 0.078961 & 0.921039 \\
\hline 3 & 0.625 & 0.390625 & 0.609375 \\
\hline 4 & 0.865 & 0.748225 & 0.251775 \\
\hline 5 & 0.481 & 0.231361 & 0.768639 \\
\hline 6 & 0.486 & 0.236196 & 0.763804 \\
\hline 7 & 0.711 & 0.505521 & 0.494479 \\
\hline 8 & 0.601 & 0.361201 & 0.638799 \\
\hline
\end{tabular}

Tabla 2. Vista de los pesos factoriales, comunalidades y unicidades 


\begin{tabular}{|l|l|}
\hline ALPHA ORDINAL & 0.833 \\
\hline
\end{tabular}

Figura 1. Vista del Alfa Ordinal

Para el coeficiente Theta de Armor el panorama es similar, ya que solo hace falta conocer el número de ítems y el mayor de los valores propios (Eigen value) para colocarlos en la hoja de cálculo (Figura 2) y llegar al coeficiente (Figura 3).

\section{CALCULO DE COEFICIENTE THETA}

\begin{tabular}{|l|l|l|}
\hline № de Items & 36 \\
\hline Valor Eigen del primer factor & 5.4 \\
\hline
\end{tabular}

Figura 2. Presentación de módulo de cálculo del coeficiente Theta

$$
\begin{array}{l|l}
\text { THETA } & 0.84
\end{array}
$$

Figura 3. Vista del coeficiente Theta

Para probar su uso se utilizaron los datos provenientes del trabajo de Elosua \& Zumbo (2008), los cuales se muestran a continuación (Tabla 3), y fueron encontrados resultados aproximados del Alfa Ordinal de dicho estudio al hallado en la herramienta propuesta en el presente trabajo (diferencias de 0.001 a favor de esta).

Tabla 3. Estimación de la confiabilidad del alfa ordinal

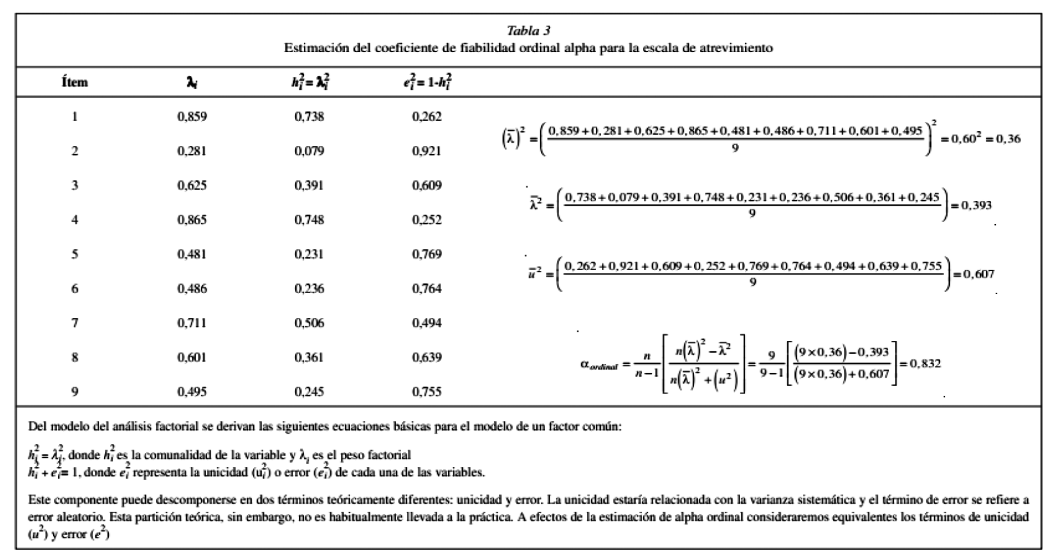

Adaptado de Elosua \& Zumbo (2008)

\section{USOS}

De acuerdo con Elosua \& Zumbo (2008), ambos coeficientes de confiabilidad son importantes, ya que obedecen a diferentes objetivos dentro de la investigación. Por un lado, si el investigador desea realizar un análisis factorial, la opción es el alfa ordinal; si solo desea estimar la varianza de las variables estudiadas, el coeficiente Theta será su mejor opción. 
Entre sus ventajas resalta que corrigen el sesgo negativo producto del procedimiento del alfa tradicional, es decir, recuperan aquellos aspectos que no fueron tomados en cuenta. Además de ello, al basarse en las variables continuas que subyacen a las respuestas que se observan, se puede considerar como un indicador de validez, ya que considera la relación entre las respuestas del ítem y la variable latente (Elosua \& Zumbo, 2008).

\section{LIMITACIONES DE LA HERRAMIENTA}

$\mathrm{Al}$ estar elaborada en Excel, la principal limitación de la herramienta es que para que pueda ser utilizada, debe estar instalado Microsoft Office en el computador. Ante ello, se recomienda a los investigadores interesados ampliar el alcance de esta herramienta a otros formatos, como el Visual Basic, o alguna estrategia de programación que sea de mayor difusión y alcance.

\section{REFERENCIAS BIBLIOGRÁFICAS}

Alarcón, R. (1998). Nuevos modelos en la medición psicológica. Lima: Universidad de San Martín de Porres, Facultad de Ciencias de la Comunicación, Turismo y Psicología.

Campo-Arias, A. \& Oviedo, H. (2008). Propiedades psicométricas de una escala: la consistencia interna. Revista de Salud Pública, 10(5), 831-839.

Cohen, R., \& Swerdlik, M. (2001). Pruebas y evaluación psicológicas: Introducción a las pruebas y a la medición (4ta ed.). México: McGraw-Hill.

Elosua, P. \& Zumbo, B. (2008). Coeficientes de fiabilidad para escalas de respuesta categórica ordenada. Psicothema, 20(4), 896-901.

Hogan, T. (2004). Pruebas Psicológicas: Una Introducción Práctica. México: Manual Moderno.

Lorenzo-Seva, U. \& Ferrando, P. (2007). FACTOR: A computer program to fit the exploratory factor analysis model. University Rovira y Virgili.

Lozano, L., García-Cueto, E. \& Muñiz, J. (2008). Effect of the number of response categories on the reliability and validity of rating scales. Methodology, 4(2), 73-79.

Muñiz, J. (1994). Teoría Clásica de los Tests. Madrid: Ediciones Pirámide.

Oviedo, H. \& Campo-Arias, A. (2005). Aproximación al uso del coeficiente alfa de Cronbach. Revista colombiana de psiquiatría, 34(4), 572-580.

Prieto, G. \& Delgado, A. (2010). Fiabilidad y validez. Papeles del psicólogo, 31(1), 67-74.

Weng, L. (2004). Impact of the number of response categories and anchor labels con coefficient alpha and test-retest reliability. Educational and Psychologycal Measurement, 64, 956-972. 
